defect in steatorrhoea, and in this issue A. G. E. Pearse and E. O. Riecken demonstrate beautifully the value of histochemical and cytochemical techniques in applied research, allowing us to make assumptions about the functions of particular organelles and to suggest changes in the interrelationship between one organelle and another.

There are also chapters on the absorption of carbohydrates, proteins, fats, salt and water, vitamins, and iron, all of which are authoritative. It is exciting, too, to learn how new techniques have thrown light on the means of transfer of these products from the intestinal lumen to the blood or lymph. The absorptive capacity of the small bowel is probably not static. It has a large functional reserve, which normally is seldom required, and $\mathbf{R}$. $H$. Dowling shows that under stress it may respond by enhancing its absorptive capacity. Increased absorption has been found after a high-bulk diet, intermittent starvation, and in malignant disease. Resection of the small bowel in man may be followed by mucosal hypertrophy and a resultant increase in the absorptive surface area. These changes were more obvious in the ileum after proximal resection than in the jejunum when the distal small bowel was removed.

Lest readers should regard this symposium as of interest to gastroenterologists only, M. D. Milne compares defects of intestinal transport with their renal counterpart, for the mechanism of absorption of some substances by the intestine is similar to that in the renal tubules. Practical clinical implications for the general physician are further expanded by Soad Tabaqchali and C. C. Booth in reviewing the significance of intestinal flora and of the antibiotic response in patients with anatomical abnormalities. Malabsorption of vitamin $B_{12}$ and fat can usually be corrected within three or four days of starting treatment with a tetracycline in a patient with, say, jejunal diverticulosis. After antibiotic treatment the bacterial count in the jejunal fluid may be expected to fall, faecal fat and nitrogen become normal, absorption of vitamin $B_{12}$ improve, and indicanuria to be much reduced. The level of indicanuria reflects indole production in patients with a stagnant loop of bowel. Increased intestinal bacterial activity acts on the amino-acid tryptophan to produce indole. Measurement of the urinary indican may therefore be used as a gross indication of indole-producing bacteria in the gut, such as Escherichia coli and Bacteroides.

\section{Premature Rupture of Membranes and Maternal Death}

With a maternal mortality of 2.58 per 10,000 total births childbearing in Britain is now safer than ever before. The need to recognize those patients at increased risk in time to take necessary precautions has been emphasized in each Maternal Mortality Report." Many recurring " avoidable factors" have been identified, but nevertheless nearly $40 \%$ of all maternal deaths are still associated with them.

It is well known that uterine sepsis can be a complication of rupture of the membranes. The identification of pathways of exogenous infection, the discovery of chemotherapeutic and antibiotic preparations, and dramatic reductions in the number of deaths from puerperal infection have contributed to a widely held but erroneous view that sepsis is no longer a serious obstetrical hazard. Its lethal role in abortion and caesarean section, particularly after prolonged labour, has been emphasized ${ }^{1}$ and evidence relevant to its association with premature rupture of the membranes has now been produced by G. A. Webb. ${ }^{2}$ His conclusions are based on an analysis of 1,054 maternal deaths reviewed by the Maternal Mortality Committee of California. Fifty-three women died after spontaneous rupture as defined by N. J. Eastman and L. M. Hellman : " rupture before the onset of labour." One death followed surgical induction. Eighteen died between the 16th and 30th week of gestation, ten by the 20th week. Though it is difficult to exclude interference in any abortion series cases were omitted if it was confirmed or even suspected. The ages of the women who died ranged from 18 to over 40 ; the highest incidence was among gravidae four-plus. Only 11 infants survived of 31 delivered after the 36th week.

Some important questions remain unanswered in Webb's paper, but there are features which merit attention. During the period of eight years premature rupture of membranes was associated with $5 \%$ of maternal deaths, and in this group sepsis was responsible in $70 \%$. Escherichia coli was the most commonly identified organism; there were only three anaerobic infections, two due to streptoccocci and one to Clostridium welchii. Twenty-two women died from septic shock, but treatment led to fatal pulmonary embolism in six and acute congestive heart failure in one, emphasizing the need for constant supervision in the care of these patients.

Dangers increased with the time interval between rupture of membranes and either delivery or the onset of pyrexia, but this interval was less than 24 hours in 15 cases. Most patients were apyrexial on admission to hospital, and Webb suggests that the systemic response to uterine infection usually occurs after the onset of labour, unless infection is overwhelming. Antibiotics were given too late and in inadequate doses. A surprising feature was that seven mothers with ruptured membranes died of amniotic embolism (four were infected) and in six this followed the administration of Pitocin or Syntocinon (both oxytocin injections). The apparent association of oxytocins and embolism justifies further study.

Premature rupture of membranes precedes approximately $10 \%$ of labours. The risk of maternal death is small (Webb assesses it as one in 5,451), but the patient should be included in a high-risk category. Rupture may indicate a complication such as malpresentation, posterior position, placenta praevia, or disproportion, but should itself be regarded as an emergency requiring specialist care. Opinions differ on whether immediate antibiotic therapy is necessary, but if given it should be designed to deal with both coliform and coccal infections. Cervical culture for organisms and sensitivity is a wise precaution at the first examination and a rising pulse rate, pyrexia, hypotension, oliguria, or icterus are definite indications for intensive antibiotic treatment. If on admission the obstetrical position is otherwise favourable a carefully controlled oxytocic infusion is indicated, but if progress is unsatisfactory or if the position is unfavourable (large foetus, high head, posterior position and uneffaced cervix) caesarean section is indicated and made safer by avoiding delay.

Premature rupture of membranes should not kill the mother or the child, but the treatment which they subsequently receive may do so.

- Mintstry of Health Report on Confidential Enquiries into Maternal Deaths in England and Wales (Reports on Public Health and Medical Subjects, No. 97 (1957), No. 103 (1960), No. 108 (1963), and No. 115 (1966) ). H.M.S.O., London.

- Webb, G. A., Amer. Y. Obstet. Gynec., 1967, 98, 594.

" Eastman, N. J., and Hellman, L. M., Williams Obstetries, 1961, 12th ed., p. 432. New York. 